\title{
Accès aux ressources biologiques de Madagascar : ambiguïtés juridiques pour les détenteurs de connaissances traditionnelles
}

Saholy Rambinintsaotra

\section{(2) OpenEdition \\ Journals}

Édition électronique

URL : http://journals.openedition.org/elohi/760

DOI : 10.4000/elohi.760

ISSN : 2268-5243

Éditeur

Presses universitaires de Bordeaux

\section{Édition imprimée}

Date de publication : 1 janvier 2014

Pagination : 117-149

ISBN : 979-10-300-0021-4

ISSN : 2431-8175

Référence électronique

Saholy Rambinintsaotra, «Accès aux ressources biologiques de Madagascar : ambiguïtés juridiques pour les détenteurs de connaissances traditionnelles », ELOHI [En ligne], 5-6 | 2014, mis en ligne le 01 janvier 2015, consulté le 21 avril 2019. URL : http://journals.openedition.org/elohi/760 ; DOI : 10.4000/elohi.760 


\section{Accès aux ressources biologiques de Madagascar : ambiguïtés juridiques pour les détenteurs de connaissances traditionnelles}

\section{SAHOLY RAMBININTSAOTRA}

Université d'Antananarivo

\section{Introduction}

Madagascar est réputé pour les richesses de sa biodiversité. Ladite biodiversité désigne l'ensemble des formes de vie : la diversité génétique (variations génétiques au sein des espèces), la diversité des espèces (végétales et animales) et la diversité de l'écosystème (variété des écosystèmes tels que les forêts tropicales, les récifs coralliens et les déserts présents sur notre planète). L’homme fait lui aussi partie de cette biodiversité, et il dépend de la diversité de la vie. La notion de biodiversité est apparue dans les années 1980 lorsqu'on s'est rendu compte de l'importance de ses valeurs pour le développement économique et social, et que cette richesse était en péril du fait des activités humaines².

La Convention sur la Diversité Biologique (CDB), mise en vigueur en décembre 1993 et ratifiée par Madagascar en 1995³, a donné aux gouvernements

1. L'expression biological diversity a été inventée par Thomas Lovejoy en 1980 tandis que le terme biodiversity lui-même a été inventé par Walter G. Rosen en 1985 lors de la préparation du « National Forum on Biological Diversity » organisé par le « National Research Council » en 1986 ; le mot biodiversité apparaît pour la première fois dans une publication en 1988 lorsque l'entomologiste américain E. O. Wilson en fait le titre du compte rendu de ce forum. Ce dernier en donne la définition suivante : " la totalité de toutes les variations de tout le vivant ». Le succès de ce néologisme tient au fait qu'il a symbolisé l'émergence de plusieurs nouveaux regards sur cette diversité du vivant.

2. Pour une information plus détaillée de cet historique, voir le site http://www.citesciences.fr/actu/dossiers/D98_1_biodiversite/html/dossier3.html

3. Décret n 95-695 du 3 Novembre 1995. 
nationaux le pouvoir de déterminer et de réglementer l'accès aux ressources génétiques. Un des trois piliers de ladite Convention est " le partage juste et équitable des bénéfices résultant de l'utilisation des ressources génétiques ". Lapplication du principe de partage des avantages issus de l'accès aux ressources génétiques s'explique par le fait que ces ressources représentent une valeur considérable. Cette valeur est liée aux possibilités d'exploitation industrielle de ces ressources (Aubertin et al, 2007). Par ailleurs, ce partage des avantages doit particulièrement contribuer à la conservation de la biodiversité ${ }^{4}$. La biodiversité d'un pays est donc considérée comme un potentiel qu'il est possible de valoriser en facilitant son accès aux différents chercheurs, aussi bien nationaux que ceux des pays industrialisés.

Dans le cadre de ce partage juste et équitable des avantages, la CDB réunit les pays riches en diversité biologique qui sont des pays en développement, et les pays riches en biotechnologie $e^{5}$, généralement les pays développés. D’une manière générale, les premiers sont appelés pays d’origine ou fournisseurs des ressources génétiques. Malgré la reconnaissance de leurs droits souverains sur les ressources de leur territoire (CDB, article 15.1), ces pays doivent s'efforcer de faciliter l'accès à leurs ressources génétiques. Les seconds, qui sont qualifiés d'utilisateurs des ressources, sont souvent des centres de recherche, des firmes agroindustrielles et biotechnologiques qui, en contrepartie de l'accès, doivent partager les avantages issus de l'exploitation desdites ressources avec l'État d'origine. Ce sont habituellement dans le cadre des industries pharmaceutiques, cosmétiques ou agricoles que ces utilisateurs exploitent ces ressources (Newman et al, 2012).

Les produits issus de ces ressources, qui sont généralement inspirés des techniques et savoirs des communautés locales, étaient libres d'accès depuis des siècles. Toutefois, les pays fournisseurs, et particulièrement les détenteurs des connaissances traditionnelles ne tirent pas profit, de façon convenable, des avantages tirés de l'accès et de l'exploitation de ces ressources (Costes, 2008).

Ainsi, ce sont surtout les pays en développement qui ont milité pour l'ajout de dispositifs d'APA (Accès et Partage des Avantages) à la CDB, et ce afin de garantir le partage des avantages découlant de l'utilisation des ressources

4. En ce sens, le document publié par l'OCDE intitulé "Préserver la diversité biologique » énonce que : « Si les espèces sauvages et leurs habitats ont une valeur négociable, les personnes ou collectivités disposant de droits de propriété exécutoires sont incitées à les protéger et à les ménager, garantissant ainsi l'existence de populations saines » (Organisation de Coopération et de Développement Économique 142).

5. La CDB définit la biotechnologie au sens large comme " toute application technologique qui utilise des systèmes biologiques, des organismes vivants ou des dérivés de ceux-ci pour réaliser ou modifier des produits ou des procédés à usage spécifique ». (Article 2 troisième alinéa). 
génétiques (Newman et al, 2012). Selon l'article 15 de la CDB, les États ont droit de souveraineté sur leurs ressources naturelles et ont donc le pouvoir de déterminer l'accès aux ressources génétiques sous leur juridiction ${ }^{6}$. Cette souveraineté de l'État sur ses ressources naturelles lui permet de procéder aux formalités juridiques d'accès et de partage des avantages. Toutefois, les formulations relatives aux processus de l'APA stipulées dans cette Convention restent vagues et relativement peu contraignantes ${ }^{7}$. Ce Traité ne se prononce pas non plus sur les mécanismes juridiques conventionnels existants à utiliser pour parvenir au partage des avantages.

C'est ainsi que, lors de la dixième Conférence des Parties à la $\mathrm{CDB}^{8}$, le Protocole de Nagoya sur l'accès aux ressources génétiques et le partage juste et équitable des avantages découlant de leur utilisation relatif à la convention sur la diversité biologique (ci-après le " Protocole ») a été adopté. Ce Protocole a pour objectif principal de i) Établir un climat de confiance réciproque entre les utilisateurs et les fournisseurs ; ii) Fixer un cadre juridique plus précis permettant de garantir le mécanisme d'APA ; iii) Assurer la sécurité juridique et l'accès à la justice des parties au contrat; iv) Inciter les fournisseurs à orienter les avantages vers la conservation de la biodiversité.

Parmi les principales innovations apportées par le Protocole, on peut noter, entre autres, la définition qu'il a donnée de "l'utilisation des ressources génétiques » comme "les activités de recherche et de développement sur la composition génétique et/ou biochimique de ressources génétiques, notamment par l’application de la biotechnologie $[\ldots] »$. En énumérant les utilisations qui

6. La souveraineté nationale est un concept qui permet de garantir que seul le gouvernement souverain peut légiférer relativement aux sujets et individus à l'intérieur de ses frontières et sous sa juridiction.

7. En effet, la plupart des dispositions de la convention commencent par les termes : « Chaque partie contractante, dans la mesure du possible et selon qu'il conviendra,...". Il en est de même pour les dispositions de son article 15 paragraphes 2, qui touchent particulièrement l'APA : "Chaque partie contractante s'efforce de créer les conditions propres à faciliter..." .

8. La Conférence des Parties (CdP, COP en anglais pour Conference of Parties) est l'instance décisionnelle qui débat tous les deux ans du fonctionnement de la CDB, des programmes de travail et de leur mise en œuvre.

9. Cf. Caillaux \& Ruiz, 2002, qui affirment que le cadre juridique de l'accès « doit être parfaitement clair sur le plan de la définition, notamment pour ce qui concerne le contenu notionnel/sémantique du vocable 'accès'. Le régime juridique qui gouverne l'accès doit en définir, sans ambiguïté, la portée et les délimitations. (Est-ce qu'il s'applique aux plantes médicinales ? à la recherche taxonomique? applique-t-on les mêmes règles aux nationaux et aux étrangers ?) La réussite d'un régime d'accès dépendra largement de ces variables » (cité par Cabrera Medaglia et López Silva, 2008). 
doivent être soumises aux règles d'APA, le Protocole a levé le doute sur la différence entre "l'acquisition normale des ressources biologiques" et "l'accès aux ressources génétiques ». En effet, «l'utilisation prévue est la clé de voûte lorsqu'une personne demande l'accès aux ressources génétiques » (Cabrera Medaglia et al, 2008). Cette définition de l'utilisation est particulièrement importante pour les populations locales car elle permet de lever l'incertitude sur les activités couvertes par la législation d'accès et celles qui en sont exclues.

Pour ce qui est du respect des règles par les utilisateurs, le traité fait obligation à toutes les parties signataires du Protocole de prendre des mesures visant à garantir que seules les ressources génétiques et les connaissances traditionnelles associées à ces ressources acquises légalement sont utilisées sous leur juridiction. Les parties doivent contrôler le respect des règles par les utilisateurs sous leur juridiction et désigner un ou plusieurs points de contrôle à cette fin. Elles doivent également prendre des mesures appropriées, efficaces et proportionnées pour régler les situations de non-respect, par les utilisateurs sous leur juridiction, des obligations qui incombent à ces derniers en matière d'accès et de partage des avantages. Les parties s'assurent également que les différends liés à des contrats spécifiques de partage des avantages puissent être portés en justice.

Un des points forts de ce Protocole est qu'il prévoit des dispositions importantes visant la participation effective des communautés autochtones et locales dans la procédure d'APA. À cet égard, ce traité : i) assimile la ressource génétique et la connaissance traditionnelle associée, sur un même pied dégalité ;ii) prend note, dans son préambule, de la Déclaration des Nations Unies sur les droits des peuples autochtones, en lien avec les droits des communautés autochtones sur les ressources ; iii) garantit la participation des communautés pour autoriser l'accès à leurs connaissances traditionnelles. Lorsque cela est prévu par la législation nationale, le Protocole prévoit l'implication des communautés en cas de demande d'accès à des ressources leur appartenant (ressources se trouvant par exemple sur des terres de statut coutumier) ; iv) prévoit que les États prennent en compte les lois coutumières des communautés dans le processus d'APA, si ces lois sont reconnues par les législations nationales.

En ce qui concerne l'accès aux ressources génétiques, l'article 6 du Protocole oblige les pays fournisseurs qui décident d'exiger un consentement préalable en connaissance de cause à assurer la sécurité juridique, la clarté et la transparence de leur législation nationale en matière d'APA et à définir des règles et des procédures claires pour l'exigence et la définition de conditions convenues d'un commun accord.

Par rapport à toutes ces dispositions, le Protocole exige donc la création ou la précision d'une législation nationale détaillée en matière d'APA, comme condition préalable de mise en œuvre effective de ce Traité. 
Ce Protocole devrait entrer en vigueur en $2014^{10}$ et Madagascar l'a ratifié en décembre 2013, ce qui veut dire que l'État s'engage à respecter ce Traité qui devient obligatoire pour lui ${ }^{11}$.

La mise en œuvre, au niveau national, de ce Protocole revêt une importance particulière et représente des défis à relever aussi bien pour le pays que pour les communautés locales concernées. Toutefois, en cas d'accès à ses ressources biologiques, Madagascar ne dispose pas encore de feuille de route, ni de cadre juridique précis définissant les droits du pays, particulièrement ceux des communautés locales " gardiens de la biodiversité " et ceux qui détiennent les connaissances traditionnelles rattachées à ces ressources (Dossou-Yovo, 2008). Il en est ainsi notamment des conditions spécifiques de la collecte et de l'exploitation des ressources biologiques, ainsi que du partage des bénéfices qui en découlent, tels que définis par le Protocole de Nagoya. En l'état actuel, l'accès à ces ressources se base essentiellement sur des contrats de bioprospection.

D'ici la mise en place du dispositif légal sur l'APA, la question qui se pose est alors de savoir quelles sont les bases juridiques sur lesquelles les différentes parties prenantes peuvent s'appuyer dans la conclusion de ces contrats de bioprospection. En d'autres termes, l'état actuel de la législation permet-il une équité des échanges entre les utilisateurs et Madagascar en tant que fournisseur des ressources génétiques ? Les contrats qui étaient conclus depuis intègrentils les dispositifs APA et dans quelles mesures contribuent-ils à la réalisation des objectifs du développement durable, particulièrement aux intérêts culturels et socioéconomiques des communautés locales et des détenteurs des connaissances traditionnelles?

Pour répondre à ces questions, une réflexion sur les forces et faiblesses du dispositif juridique existant, ayant trait au processus APA, va être abordée. Par la suite, pour illustrer les difficultés de la mise en œuvre d'une « équité » dans le partage des avantages à Madagascar, un exemple de contrat de bioprospection va être présenté. Enfin, des marges d'améliorations possibles pour une mise en œuvre effective du protocole, surtout au niveau des communautés locales et des détenteurs des connaissances traditionnelles concernées, vont être proposées.

10. Lors de la récente onzième Conférence des Parties sur la CDB, qui s'est tenue en Inde au mois d'octobre 2013, 28 pays africains sur 98 ont signé ce nouveau Protocole. Madagascar, en tant que pays fournisseur des ressources génétiques, a signé ce Protocole en novembre 2011 et, de ce fait, s'est engagé à œuvrer pour sa mise en œuvre et sa ratification.

11. Dans les pays de droits de tradition civiliste comme Madagascar, un Traité conclut par l'État devient partie du droit interne de cet État dès lors que celui-ci a exprimé son consentement à être lié par la ratification de ce Traité. Les droits et obligations du droit international peuvent découler directement de ce Traité. 


\section{État des lieux de quelques mesures juridiques nationales contribuant à la mise en œuvre du Protocole}

Parmi les innovations apportées par le Protocole de Nagoya figure l'obligation pour les pays fournisseurs des ressources génétiques de promulguer une loi ou un règlement imposant l'obtention de son consentement préalable en connaissance de cause pour acquérir ses ressources génétiques.

Le Protocole ajoute donc une obligation qui nest pas prévue par la CDB. Ainsi, les pays fournisseurs qui nélaborent aucune loi ou réglementation spécifique pour régir l'accès aux ressources génétiques ne pourront pas exiger des pays utilisateurs qu'ils adoptent des mesures de mise en conformité.

Pour le cas de Madagascar, comme on l'a signalé précédemment, le cadre juridique spécifique au Protocole fait défaut. Un avant-projet de loi sur l'accès aux ressources biologiques est en cours d'élaboration depuis une dizaine d'années. Il a fait l'objet de plusieurs refontes mais, à ce jour, il n'a pas encore été adopté. En tant que fournisseur de ressources génétiques, le pays ne doit plus attendre des années pour promulguer un cadre juridique spécifique à l'APA car ce retard favorise le «néocolonialisme vert à Madagascar» (Hufty et al, 1995).

Il convient de signaler que, même en l'absence de dispositifs spécifiques à l'APA, des contrats de bioprospection ${ }^{12}$ (Cabrera Medaglia et al, 2008) ont été signés à Madagascar. Ces derniers sont généralement le fruit d'une collaboration entre les instituts de recherche malgaches et des laboratoires étrangers. Faute d'avoir accès au détail du contenu de tels contrats, les questions qui se posent sont alors de savoir sur quelles bases juridiques ceux-ci ont été signés et dans quelles mesures les connaissances traditionnelles malgaches associées aux accès ont été valorisées économiquement au bénéfice de leurs détenteurs/découvreurs. Ces questions sont posées car, après enquêtes, certains utilisateurs prétendent avoir respecté les lois en vigueur à Madagascar. Ainsi, pour essayer de répondre à ces questions, quelques textes juridiques obéissant à certains critères des dispositifs APA vont être présentés brièvement.

\section{Fondements juridiques d'une utilisation durable des ressources} naturelles

Dans le cadre de l'utilisation durable des ressources naturelles, la Charte de l'environnement malgache (Loi $\mathrm{n}^{\circ}$ 90-033 modifié) constitue le cadre général d'exécution de la politique nationale de l'environnement. Toutefois, ce texte ne contient aucune disposition spécifique qui reflète le partage équitable des avan-

12. La bioprospection consiste en la recherche parmi les organismes présents dans la nature de nouveaux produits pouvant présenter un intérêt pour les industries agricole et surtout pharmaceutique. 
tages découlant de l'accès aux ressources génétiques/biologiques malgaches et des connaissances traditionnelles associées.

Par ailleurs, la mise en compatibilité des investissements avec l'environnement (décret $\mathrm{n}^{\circ}$ 99-954 modifié) fait obligation à tout demandeur de permis de collecte et de vente d'espèces destinées à l'exportation de faire une étude d'impact environnemental et social. Il en est de même pour toute collecte et/ ou chasse et vente d'espèces n'ayant jamais fait l'objet de commercialisation par le passé. À la suite d'une évaluation sociale et environnementale favorable, un permis environnemental est délivré au profit dudit promoteur. Ce permis constitue un préalable obligatoire à tout commencement des travaux de collecte de ressources naturelles à Madagascar. Toutefois, il importe de noter que le permis environnemental est tout à fait différent du permis autorisant la collecte et la prospection génétique dite bioprospection (Moretti et al, 2007). Une étude d'impact environnemental est une procédure qui a pour objet de s'assurer qu'une information rapide et adéquate a été obtenue sur les conséquences éventuelles sur l'environnement de projets de développement, sur les alternatives possibles et sur les mesures pour réduire les dommages (Shelton, 1999). Il ne donne pas lieu à un partage des avantages au sens de l'article 15 de la CDB et du Protocole de Nagoya. En effet, dans l'esprit de la CDB et du Protocole, l'accès aux ressources génétiques englobe les aspects suivants : une autorisation préalable des autorités compétentes à tous les niveaux ; l'existence de procédures de négociations de conditions convenues d'un commun accord (CCCA) et le partage juste et équitable des avantages (au moyen de contrats), et l'exigence que les CCCA prévoient des mécanismes de traçage et de surveillance (Cabrera Medaglia \& López Silva, 2007).

Madagascar dispose également d'une législation portant sur la mise en œuvre de la Convention sur le commerce international des espèces de faune et de flore sauvages menacées d'extinction de 1973 - également appelée " convention CITES », et ce, à travers la loi no $2005-018$ du 17 octobre 2005 sur le commerce international des espèces de faune et de flore sauvages et ses textes subséquents d'application. Cette loi soumet à autorisation l'exportation des espèces animales et végétales figurant dans ses annexes. C'est le ministère chargé des forêts qui est l'organe de gestion de la CITES. Il est le seul habilité à délivrer les permis et autorisations de sortie des espèces. Le Ministère chargé de la recherche scientifique en est l'autorité scientifique. La convention CITES est complémentaire avec le protocole de Nagoya dans la mesure où les ressources objets de demande d'accès font parties de celles qui sont visées par la Convention CITES.

Toujours dans le cadre de l'autorisation de collecte, l'arrêté ${ }^{\circ}$ 6686/000 du 4 juillet 2000 réglementant l'exploitation et la commercialisation des produits accessoires des forêts permet l'usage économique durable des forêts. Ce texte 
définit comme produits accessoires des forêts, les plantes médicinales utilisées principalement en médecine et pour la préparation de produits pharmaceutiques et chimiques (Drosera, Centella, Catharanthus, Rauwolfa, Pygeum, etc.), ainsi que les plantes vertes des essences forestières, les extraits et huiles essentielles des essences forestières, etc. Ces produits ne peuvent être collectés ou exploités sans convention ou permis d'exploiter ou sans attestation d'un producteur privé. Accordées pour une durée maximale de deux ans, ces autorisations ne sont ni cessibles ni transmissibles. Tout récolteur et collecteur de produits accessoires de forêts ne peut exercer cette fonction qu'en vertu du mandat d'un exploitant forestier titulaire d'une convention ou d'un permis valide. Ladministration forestière se réserve le droit de contingenter ou d'interdire leur exportation à l'état brut tant que la capacité de production pérenne n’est pas assurée.

D’après nos enquêtes, les bioprospecteurs se basent uniquement sur les conditions juridiques de cette autorisation de collecte pour l'accès et l'exportation des ressources génétiques forestières.

Or, tout comme le permis environnemental, délivré à la suite d'une évaluation favorable de l'environnement, et le permis d'exportation basé sur la CITES, l'autorisation de collecte ne peut pas être assimilé au permis d'accès aux ressources génétiques tel que défini par les dispositions de l'article 15 de la CDB et du Protocole de Nagoya. Ces derniers requièrent un processus particulier nécessitant le partage équitable des avantages découlant de l'utilisation desdites ressources et des connaissances traditionnelles associées ${ }^{13}$. Dans la perspective d'une demande d'accès aux ressources génétiques telle que définie par le Protocole de Nagoya, ces trois types de permis sont complémentaires, et peuvent lui servir de bases juridiques.

\section{Droits des communautés locales utilisables pour organiser le régime juridique de l'APA}

En matière d'APA, la connaissance des droits de propriété et d'occupation sur les ressources génétiques est très importante. En effet, les utilisateurs des ressources génétiques doivent s'assurer qu'un fournisseur est bien autorisé à fournir de telles ressources. Souvent, cette habilité n’incombe pas uniquement à

13. À sa huitième réunion (du 7 au 11 octobre 2013 à Montréal) le Groupe de travail de la CDB sur l'article 8 (j) (connaissances traditionnelles) et les dispositions connexes, a adopté un projet de plan d'action pour l'utilisation coutumière durable, et a recommandé lélaboration de directives sur le rapatriement, le consentement préalable donné en connaissance de cause par les communautés autochtones et locales pour l'accès aux connaissances traditionnelles, le partage des avantages qui en découlent, l'établissement des rapports et la prévention de l'appropriation illicite de ces connaissances (Bulletin des Négociations de la Terre 9.612, 24 février 2014). 
l'État mais également à ceux qui ont des droits privés ou autres droits, tels que les droits d'occupation, sur les terres ou les ressources (Cabrera et al, 2008). Ensuite, cette précision permet de déterminer qui a le droit de participer aux processus de négociation et de prise de décision en matière d'APA, et de bénéficier de ses avantages potentiels.

En ce sens, l'article 6.2 du Protocole de Nagoya permet la participation des communautés locales aux dispositifs APA, à travers le consentement préalable en connaissance de cause de celles-ci, à condition qu'elles aient des droits établis sur les territoires où se situent les ressources génétiques objets d'accès. En outre, son article 5.2, prévoit « la nécessité de s’assurer que les avantages découlant de l'utilisation des ressources génétiques qui sont détenues par les communautés autochtones et locales, conformément au droit interne relatif aux droits établis de ces communautés autochtones et locales sur ces ressources génétiques, sont partagés de manière juste et équitable avec les communautés concernées conformément à des conditions convenues d'un commun accord ». Ainsi, le Protocole se réfère au "droit interne " pour toute reconnaissance de droits des populations locales sur les ressources objets d'accès et sur le partage des avantages issus de cet accès.

En ce sens, la loi sur le transfert de gestion locale des ressources naturelles renouvelables (loi $\mathrm{n}^{\circ}$ 96-025) constitue un outil permettant aux usagers coutumiers de participer « à la conservation durable des ressources naturelles renouvelables ${ }^{14}$, et ce, sous forme de contrat de transfert de gestion locale. Ledit contrat confère à la communauté de base bénéficiaire, " la gestion de l'accès ou le droit d'usage, de l'exploitation et de la valorisation économique et de la conservation de ces ressources transférées ${ }^{15}$. Ainsi, en transférant, sous forme de contrat, aux populations locales la gestion des ressources naturelles cette loi confie à ces populations la conservation, la préservation et la valorisation économique des ressources transférées (Blanc-Pamard et al, 2007). En tant que gestionnaires légales desdites ressources naturelles, ces populations locales peuvent donc prétendre au partage équitable des avantages, en cas d'utilisation des ressources naturelles dont elles ont la garde.

À côté de ces cadres juridiques qui peuvent servir de base pour contribuer à la mise en œuvre du Protocole, il convient de présenter brièvement quelques défis et problèmes causés par l'absence de cadre juridique spécifique à l'APA.

14. Article $1^{\text {er }}$ de la loi $n^{\circ} 96-025$ du 30 septembre 1996 sur la gestion locale des ressources naturelles renouvelables.

15. Article 43 de la loi $n^{\circ} 96-025$ sur la gestion locale des ressources naturelles renouvelables. 


\section{Problèmes et défis de mise en œuvre nationale de l'APA}

Comme on l'a déjà signalé, le cadre juridique définissant les droits du pays et ceux des détenteurs des connaissances traditionnelles associées aux ressources génétiques, en matière d'APA, fait défaut à Madagascar. En l'état actuel de la législation, l'accès aux ressources génétiques est accordé par le biais d'une législation générale décrite précédemment. Ainsi, les opérateurs désirant faire de la prospection des ressources génétiques à Madagascar ne sont pas obligés de solliciter un accord préalable, tel que défini par le processus APA, ni de s'engager à une contrepartie. Ce manque de mesures cause une incertitude juridique et limite le désir des différentes instances concernées par les ressources génétiques à s'engager dans des négociations portant sur l'accès et le partage des avantages (Buck et al, 2011). Ces insécurités et incertitudes concernent les éléments suivants.

\section{Incertitude sur les droits des détenteurs des connaissances traditionnelles associées aux ressources génétiques}

Les connaissances traditionnelles sur les plantes et leurs effets sont enracinées dans la société malgache depuis la nuit des temps. Ces connaissances sont généralement à l'origine d'études scientifiques conduites à Madagascar sur les substances naturelles dorigine végétale (Cortadellas et al, 2010 ; Péchard et $a l, 2005)$. Elles sont librement utilisées et partagées par les tradipraticiens. En Afrique, Madagascar fait partie des pays qui ont inclus des médicaments traditionnels dans leurs listes nationales de médicaments essentiels (Organisation mondiale de la Santé, 2011). Dans ce cadre, le Madeglucyl est utilisé pour le traitement des cas de diabète non compliqué (Ratsimamanga et al). Cette valorisation des plantes de Madagascar se fait, non seulement au pays, mais également à l'étranger ${ }^{16}$.

Généralement, les connaissances traditionnelles sont utilisées dans le cadre de ces types d'inventions biotechnologiques et en cas de découverte concluante, peuvent faire l'objet de demande de protection par brevet. Or, traditionnellement, les détenteurs de ces savoirs ne profitent pas des avantages qui en découlent (Fleurentin et al, 2002 ; Aubertin et al, 2007 ; Delpas, 2012). C'est le cas de la pervenche rose de Madagascar qui a servi de matière première à la constitution d'une drogue contre la leucémie et qui a généré des profits énormes pour

16. En 2010, le pays a exporté plus de 900 tonnes de plantes médicinales selon l'Institut national de la statistique malgache (Instat). Selon les informations le « Mandravasarotra " ou l'aloés (Cinnamomsa fragrans) et le Ravintsara (Cinnamomum camphora) sont les plus demandés. La Gazette de la Grande Ile, 15 mars 2014. 
un laboratoire américain, sans aucune retombée économique ni pour le pays, ni pour les détenteurs des connaissances traditionnelles ${ }^{17}$.

Dans ce cadre, le Protocole de Nagoya vise à promouvoir un partage équitable des avantages et à éviter la biopiraterie ${ }^{18}$ (utilisation de ressources génétiques originaires des pays en développement sans avoir accédé à ces ressources de façon adéquate conformément au droit international et national concerné) (Dutfield, 2004). Il impose alors aux Parties à la convention de prendre des mesures visant à partager les avantages découlant de l'utilisation de connaissances traditionnelles avec les communautés locales détentrices de ces connaissances traditionnelles (article 5, paragraphe 5). Ce partage des avantages doit être basé sur des conditions convenues d'un commun accord. Le protocole impose également aux Parties de prendre des mesures pour garantir que l'accès aux connaissances traditionnelles soit soumis au consentement préalable en connaissance de cause ou à l'accord et à la participation des communautés autochtones et locales (article 7, et 16). En ce sens, l'article 12-1 renvoie au droit interne les processus de mise en ouvre de la connaissance traditionnelle associée aux ressources génétiques. Se pose alors la question des modalités de participation des communautés locales organisées par le droit national. Cette question se rattache à la reconnaissance de ces communautés et aux droits qui leur sont reconnus.

\section{Flou du statut juridique des détenteurs des connaissances traditionnelles}

Par rapport aux détenteurs des connaissances traditionnelles associées aux ressources génétiques, l'identification desdites connaissances constitue une source d'information importante car elles permettent de fournir des indices importants pour la découverte scientifique de propriétés génétiques ou biochimiques des ressources génétiques (Johnson, 2011). Il convient toutefois de signaler que

17. Au début des années 1950, suite aux révélations de guérisseurs locaux de Madagascar, des chercheurs d'Eti Lilly Pharmaceuticals ont extrait deux puissants alcaloïdes anticancéreux de la pervenche rose : la vinblastine et la vincristine. Les ventes mondiales de ces deux substances brevetées par Eli Lilly lui ont rapporté un profit annuel de 220 millions de dollars, mais pas un centime n'est revenu à Madagascar ni aux guérisseurs. 18. La notion " piraterie biologique / biopiraterie " n'a pas de définition juridique généralement admise. Pour certains, il s'agit de l'acquisition de ressources génétiques et de connaissances traditionnelles sans le consentement du pays ou du détenteur de la ressource/connaissance; quand il n'existe pas de règles sur le partage équitable des avantages ; lorsque l'on sait que les droits de propriété intellectuel (DPI) protègent des innovations qui ne sont que des copies ou des modifications superficielles des ressources génétiques; lorsque les DPI protègent des innovations biotechnologiques basées sur des ressources génétiques, avec ou sans consentement préalable, etc. 
les notions de " connaissances traditionnelles associées à des ressources génétiques » et de "détention" de ces informations par une communauté autochtone et locale n'ont pas encore fait l'objet de définitions universellement admises (Bérard et al, 2005). Certes, les connaissances traditionnelles sont partiellement définies dans l'article 8 (j) de la CDB comme « les connaissances, les innovations et les pratiques des communautés autochtones et locales » en lien avec la conservation et l'utilisation durable de la diversité biologique ${ }^{19}$. Toutefois, les définitions internationales de ces termes et de ces concepts font actuellement l'objet de négociations au sein du comité intergouvernemental de l'Organisation mondiale de la propriété intellectuelle ${ }^{20}$.

En 2008, le comité intergouvernemental de l'OMPI a proposé de définir le "savoir traditionnel » comme un "savoir résultant d'une activité intellectuelle dans un contexte traditionnel, [qui] comprend le savoir-faire, les techniques, les innovations, les pratiques et l'apprentissage qui font partie des systèmes de savoirs traditionnels, ledit savoir s'exprimant dans le mode de vie traditionnel des communautés autochtones ou locales, ou étant contenu dans les systèmes de savoirs codifiés transmis d'une génération à l'autre. Le terme n’est pas limité à un domaine technique spécifique, et peut s'appliquer à un savoir agricole, écologique ou médical, ainsi qu’à un savoir associé à des ressources génétiques » (OMPI 2008). Dans le cadre du dispositif APA, « un lien juridique entre les communautés et les connaissances traditionnelles associées aux ressources génétiques dont elles sont détentrices est nécessaire pour permettre leur participation » (Fondation pour la Recherche de la Biodiversité, 2010).

Par rapport à cette définition, et au dispositif APA, on constate que les droits des communautés locales liés à l'utilisation de leurs connaissances traditionnelles associées aux ressources génétiques ne bénéficient pas encore de protection juridique à Madagascar. Sur le plan du droit positif, l'ordonnance $\mathrm{n}^{\circ}$ 89-019 instituant un régime pour la protection de la propriété industrielle

19. Selon l'article $8(\mathrm{j})$ de la $\mathrm{CDB}$, « chaque Partie contractante, dans la mesure du possible et selon qu'il conviendra: [... j) Sous réserve des dispositions de sa législation nationale, respecte, préserve et maintient les connaissances, innovations et pratiques des communautés autochtones et locales qui incarnent des modes de vie traditionnels présentant un intérêt pour la conservation et l'utilisation durable de la diversité biologique et en favorise l'application sur une plus grande échelle, avec l'accord et la participation des dépositaires de ces connaissances, innovations et pratiques et encourage le partage équitable des avantages découlant de l'utilisation de ces connaissances, innovations et pratiques $"$.

20. L'OMPI rédige actuellement des projets d'articles sur la protection des CT (voir le chapitre 3.2) disponible à l'adresse: http://www.wipo.int/edocs/mdocs/tk/en/wipo_grtkf_ic_19/wipo_grtkf_ic_19_5.pdf. 
à Madagascar et son texte d'application réglementent, selon des conditions communément fixées, les demandes, l'octroi et la protection des brevets et des certificats d'auteurs d'inventions, de marques de fabrique ou de commerce, des dessins et des modèles industriels et des noms commerciaux. Toutefois, ce texte ne contient aucune disposition relative aux connaissances traditionnelles et à l'appropriation illicite ou à une utilisation abusive par un tiers de celles-ci (Comité intergouvernemental de la propriété intellectuelle relative aux ressources génétiques, aux savoirs traditionnels et au folklore, 2010). Ce texte interdit également la brevetabilité des variétés végétales et animales, des procédés essentiellement biologiques d'obtention des végétaux ou d'animaux et des produits pharmaceutiques (plantes médicinales). Pour être conforme au cadre international sur la propriété intellectuelle, et particulièrement sur le système des brevets, il fait actuellement l'objet d'une refonte.

Parmi les innovations apportées, et touchant particulièrement au processus APA, on peut citer, entre autres, lobligation pour les demandeurs de brevet, de divulguer le nom du pays fournissant les ressources biologiques et/ou connaissances traditionnelles associées, lorsque la demande de brevet concerne des ressources biologiques et/ou des connaissances traditionnelles associées. En procédant de la sorte, les avantages issus des ressources génétiques peuvent être identifiés et contrôlés (Ruiz \& Lapena, 2007). En ce sens, la divulgation de la source est liée à la traçabilité, et particulièrement dans le système de brevets ${ }^{21}$. Mais le problème est de savoir si cette divulgation permet réellement le partage des avantages. Dans ce cadre, le Protocole de Nagoya donne beaucoup plus de précisions en affirmant que les éventuels utilisateurs des ressources génétiques et des savoirs traditionnels devront obtenir le consentement préalable libre et éclairé des communautés détentrices de ces savoirs, avant d'y accéder et de les utiliser. Le protocole n'impose pas de divulguer l'origine des ressources génétiques dans les demandes de brevets, comme le demandaient les pays en développement, mais impose aux parties de désigner des " points de contrôle " (Buck \& Hamilton, 2011). Dans ce cadre, il convient de signaler que lors de la 26e session du Comité intergouvernemental de l'OMPI sur la propriété intellectuelle relative aux ressources génétiques, aux connaissances traditionnelles et au folklore du 3 au 7 février 2014 à Genève, des dispositions relatives à l'inclusion de la divulgation obligatoire de l'origine des ressources génétiques utilisées par les demandeurs de brevets, ainsi qu'une gamme de sanctions après la délivrance

21. En ce sens, voir Document WIPO/GRTKF/IC/9/8 sur la reconnaissance des savoirs traditionnels dans le système des brevets : projet intérimaire, du 15 mars 2006, analysé dans la neuvième session du Comité intergouvernemental de l'OMPI, Genève, 24-28 avril 2006. 
pour le non-respect de l'obligation de divulgation, restent en suspens (Bulletin des Négociations de la Terre, 2014).

En outre, ce projet de refonte prévoit également l'obligation pour les demandeurs de brevet sur les ressources génétiques, « de communiquer des éléments de preuve indiquant la conformité avec les prescriptions légales applicables dans le pays fournisseur en ce qui concerne le consentement préalable donné en connaissance de cause pour l'accès et le partage équitable des avantages découlant de l'utilisation commerciale ou autre de ces ressources et/ou connaissances traditionnelles associées $»^{22}$. Il importe toutefois de signaler que cette disposition nécessite une clarification légale d’octroi ou de refus du consentement préalable en connaissance de cause, avec les difficultés d'obtention de cette procédure, et que le législateur doit en tenir compte (Cabrera Medaglia et al, 2008).

Ensuite, la demande de brevet d'invention concernant les ressources biologiques et/ou les connaissances traditionnelles associées peut être rejetée lorsque le demandeur n'a pas respecté l'obligation de divulguer le nom du pays fournissant ces ressources et/ou l'obligation de fournir des informations et des preuves sur le principe du consentement préalable et le partage des avantages (article 8 bis). Les communautés locales ont également la possibilité de jouir " de droits intellectuels collectifs, inaliénables et imprescriptibles sur [leur] patrimoine naturel et culturel ».

Le problème dans la mise en œuvre pratique de cette obligation est que les pays utilisateurs ont toujours été réticents à procéder à la divulgation de lorigine des ressources génétiques et des savoirs traditionnels qu'ils ont eus à utiliser dans le cadre de leurs recherches et de leur demande de brevet (Greiber et al, 2012). Ils le sont aussi lorsqu'il s'agit de procéder au partage des avantages. De plus, le Protocole reste vague sur les points de contrôle et les sanctions en cas de non-respect de ses dispositions. Ainsi, en cas de manquement, il n'y a pas de sanctions assez dissuasives pour ramener les pays utilisateurs à l'ordre. Le Protocole laisse aux États le soin de prendre les mesures appropriées pour s'assurer que le consentement des fournisseurs sur la base de termes mutuellement convenus a été donné pour l'accès. Or, ni le projet de loi sur l'APA, ni ce projet de refonte de l'ordonnance instituant un régime pour la protection de la propriété industrielle ne contient de sanction à cet égard. Pour ce faire, il importe que ces textes prévoient des sanctions plus efficaces à cet effet.

En ce sens, on peut s'inspirer des travaux de l'OMPI qui rédige actuellement des projets d'articles sur la protection des connaissances traditionnelles

22. Cette disposition est conforme à celle proposée par le Comité intergouvernemental de la propriété intellectuelle relative aux ressources génétiques, aux savoirs traditionnels et au folklore de l'OMPI pour sa vingt-septième session à Genève prévue du 24 mars au 4 avril 2014. 
(OMPI, 2014). Les options de protection incluent le droit d'empêcher l'octroi de droit de propriété intellectuelle impliquant l'utilisation de connaissances traditionnelles sans la divulgation obligatoire des détenteurs de ces connaissances traditionnelles, du pays d'origine et sans preuve du respect des exigences en matière de connaissances traditionnelles et de partage des avantages. Le projet propose des dispositions relatives aux sanctions et recours en cas de violation de la protection requise des connaissances traditionnelles ainsi que la possibilité de mettre en place des autorités compétentes (au niveau régional, national ou local) chargées de défendre les détenteurs de connaissances traditionnelles. Il contient également des dispositions en vue d'un mécanisme global de partage des avantages dans le cas de connaissances traditionnelles transfrontalières pour lesquelles il n'est pas possible de donner ni d'obtenir un consentement préalable en connaissance de cause. Ce projet mentionne également la cohérence avec d'autres instruments internationaux, et en particulier avec le protocole de Nagoya ${ }^{23}$.

Absence de mécanisme de partage des avantages pour les tradipraticiens

Les activités des détenteurs des connaissances traditionnelles, et particulièrement dans le cadre de l'exercice de la médecine traditionnelle, de la pharmacopée et des plantes médicinales, sont reconnues légalement par les dispositions de la loi portant code de santé (Loi $\mathrm{n}^{\circ}$ 2011-002). Ainsi, la médecine traditionnelle y est définie comme «la somme totale de toutes les connaissances et pratiques, utilisées en diagnostic, prévention et élimination des déséquilibres physique, mental et social et reposant exclusivement sur les expériences pratiques et les observations transmises de génération en génération, oralement ou par écrit, permettant de prévenir, de guérir les maladies et d'alléger les souffrances ». Est qualifié de tradipraticien de santé « toute personne qui pratique la médecine traditionnelle ». Les tradipraticiens sont autorisés à se constituer en association chargée d'assurer la promotion et le développement de la médecine traditionnelle, à condition que celle-ci soit reconnue officiellement comme d'utilité publique (article 96).

Les documents, objets et remèdes utilisés en médecine traditionnelle doivent être présentés à toute réquisition des autorités sanitaires ou administratives des localités d'exercice ainsi quà tout contrôle ordonné par le Ministre chargé de la Santé ou par le Conseil National de la médecine traditionnelle (article 100).

La Commission de la pharmacopée traditionnelle malagasy, placée sous l'autorité du Ministre chargé de la Santé, a pour tâche de réunir les informations

23. Voir : Comité intergouvernemental de la propriété intellectuelle relative aux ressources génétiques, aux savoirs traditionnels et au folklore. Article 10. Disponible à l'adresse : www.wipo.int/edocs/mdocs/tk/fr/wipo...ic.../wipo_grtkf_ic_27_4.pdf 
concernant les plantes et autres produits naturels susceptibles dêtre utilisés en thérapeutique et de codifier leur emploi et la préparation des médicaments galéniques qui en sont faits. Cette tutelle des tradithérapeutes au système de santé constitue un moyen pour le système de santé de contrôler les activités des tradipraticiens et de prévenir les risques sanitaires liés à celles-ci (Lefèvre, 2008).

En létat actuel de la situation, 15 associations de tradipraticiens sont agréées par le Ministère de la Santé. Cependant, seuls deux organismes intégrés dans la médecine traditionnelle se sont vus octroyer des Autorisations de Mise sur le Marché : l'Institut malgache des recherches appliquées (IMRA) ${ }^{24}$ et le laboratoire pharmaceutique Homéopharma ${ }^{25}$. Cette situation s'explique par le fait que, la reconnaissance des soins par les plantes nécessite l'aval d'un laboratoire (Lefèvre, 2008). Il est à noter toutefois que ce texte reste silencieux sur les droits des communautés en cas d'accès à leurs connaissances. Aucun mécanisme permettant la rémunération des connaissances traditionnelles associées aux espèces exploitées par de telles institutions n'est prévu. Il en est ainsi, notamment, du "Centella asiatica » qui a été utilisé par la médecine traditionnelle bien avant la mise au point du Madécassol par l'IMRA. Le Madécassol est une pommade cicatrisante qui a été brevetée par les laboratoires Laroche-Navarron (Péchard et al, 2005).

Précarité du droit au foncier des détenteurs des connaissances traditionnelles

En se référant à la définition des savoirs traditionnels proposée par l'OMPI citée précédemment, on constate que ce terme ne se limite pas au domaine médical et peut s'appliquer également " aux terres", compte tenu du lien étroit entre ces dernières et les connaissances traditionnelles (Fondation de la Recherche sur la Biodiversité, 2010). Cette dépendance des connaissances traditionnelles à la terre est d'ailleurs consacrée par le droit international. Ainsi, « les gouvernements devraient reconnaître et encourager les méthodes traditionnelles et les connaissances des populations autochtones et de leurs collectivités [...] pour assurer la préservation de la diversité biologique et l'utilisation durable des ressources biologiques » (chapitre XV de l'Agenda 21). "Les populations autochtones et leurs communautés ont un lien historique avec leurs terres et sont généralement les descendants des habitants originaux de ces terres [...] Elles ont développé au cours des générations une connaissance scientifique traditionnelle et holistique de leurs terres, de leurs ressources naturelles et de

24. IMRA, organisme de recherche privé dans le secteur des substances naturelle, créé en 1957 par le Professeur Rakoto Ratsimamanga, possède plusieurs brevets productifs comme le Madécassol.

25. Homéopharma est créé en 1992 comme antenne de Boiron dont il s'est séparé en 2003. Il est spécialisé en aromathérapie et cosmétologie. 
leur environnement " (chapitre XXVI de l'Agenda 21). De même, le préambule de la Convention sur la diversité biologique insiste sur cette dépendance à travers la nécessité d'impliquer les populations dans le processus décisionnel national en vue d'une conservation intégrée ${ }^{26}$.

Par rapport à ce lien entre les connaissances traditionnelles et la terre, la Constitution malgache reconnaît ${ }^{27}$, dans son préambule ${ }^{28}$, la Charte Africaine des Droits de l'Homme et des Peuples comme partie intégrante de son droit positif. Cette charte énonce dans ses dispositions les droits de propriété des peuples locaux, propriété fondée sur leur occupation et leur exploitation traditionnelles des terres ${ }^{29}$. Cette consécration constitutionnelle est importante dans la mesure où elle permet « de guider les juges et les administrateurs dans l'accomplissement de leurs tâches, notamment lorsque la solution d'un problème ne ressort pas clairement du droit positif » (Mekouar 2001). Toutefois, la nature des droits accordés aux propriétaires coutumiers et aux usufruitiers, en cas de restriction d'accès et/ou d'accès aux ressources foncières n'est pas clairement définie par cette loi fondamentale. En effet, en droit malgache, les droits locaux fonciers sont fondés sur la propriété ou les droits d'usages (Rarijaona, 1967 ; Ramarolant, 1989 ; Muttenzer, 2010).

En ce qui concerne le droit de propriété, les textes sur le foncier ont fait l'objet d'une refonte en 2005 en vue de « répondre aux attentes de sécurisation

26. « Reconnaissant qu'un grand nombre de communautés locales et de populations autochtones dépendent étroitement et traditionnellement des ressources biologiques sur lesquelles sont fondées leurs traditions et qu'il est souhaitable d'assurer le partage équitable des avantages découlant de l'utilisation des connaissances, innovations et pratiques traditionnelles intéressant la conservation de la diversité biologique et l'utilisation durable de ses éléments » (Préambule de la Convention sur la Biodiversité de 1992).

27. La reconnaissance par la Constitution malgache de la Charte Africaine des Droits de l'Homme constitue une opportunité importante pour faire valoir les droits de l'homme à l'environnement et au foncier. En effet, compte tenu du fait que ces droits consacrés dans la plus haute hiérarchie des normes, ils sont à l'abri des lois et des majorités de circonstance qui peuvent éventuellement ignorer ces droits.

28. En ce sens, il convient de noter que les dispositions de l'article 13 de lordonnance n62-041, du 19 septembre 1962 portant dispositions générales de droit interne et de droit international privé, confèrent un caractère obligatoire aux principes généraux contenus dans le préambule de la Constitution (J.O.R.M. n 2571 du 26.04.99, p. 1060). Autrement dit, selon ce texte, le préambule de la Constitution a la même valeur que les dispositions constitutionnelles elles-mêmes.

29. Voir paragraphe $1^{\text {er }}$, article 21 de la Charte. 
foncière $»^{30}$. Cette réforme tend vers la privatisation des terres par l'enregistrement des droits et la généralisation des procédures d'immatriculation. Elle permet aux acteurs locaux de faire reconnaitre leurs droits sur leur propriété grâce à la procédure de délivrance des certificats fonciers (FIDA, 2011 ; Andrianirina, 2012). En fait, cette refonte occulte les droits des propriétaires coutumiers et augmente encore la précarité foncière des usufruitiers et des propriétaires coutumiers. En effet, l'acquisition du certificat foncier demande des moyens financiers, des pièces d'identité et des signatures non accessibles aux populations défavorisées. Par ailleurs, en létat actuel de la législation, les droits sur les ressources naturelles ne font pas encore l'objet de délivrance d'un titre de propriété à travers le certificat foncier car ils ne sont pas inscrits dans le Plan Local d'Occupation Foncière (Rambinintsaotra et al). Ainsi, l'exigence d'un titre, comme preuve de droits sur les ressources foncières, dispense ceux qui ne peuvent pas se prétendre à la titrisation foncière de se prévaloir de leurs droits au consentement et à la participation aux prises de décisions, notamment en cas d'accès à leurs ressources naturelles (Lavigne-Delville et al, 2009).

Pour ce qui est du droit d'usage, l'identification des communautés locales détentrices de ces droits sur les ressources naturelles n'est pas clairement définie par le droit positif. Dans ce cadre, ce sont principalement la loi forestière et le code des aires protégées qui en donnent les indications. Ainsi, ce sont les populations rurales riveraines des forêts qui peuvent exercer les droits d'usage qui leur ont été reconnus, soit en vue d'assurer leurs activités traditionnelles par collecte des produits forestiers secondaires, soit en vue de satisfaire leurs besoins domestiques ${ }^{31}$. On constate toutefois que les droits d'usage définis par ces textes restent vagues. En premier lieu, le texte ne précise pas la superficie et la consistance des terrains objets des droits d'usage. Ensuite, la qualification de "riveraines " mériterait dêtre précisée : à partir de quelle distance de la forêt n'est-on plus riverain ? Les riverains des riverains peuvent-ils être considérés comme riverains s'ils ont des pratiques habituelles dans la forêt en cause ? Par ailleurs, la nature des droits d'usage n'est précisée par aucun texte forestier. Enfin, il importe de signaler que ces droits d'usage permettent à ces populations d'exploiter les ressources de la forêt en vue de leur subsistance mais, nétant pas propriétaires, ces droits (en l'état actuel de la législation) ne leur permettent pas de contrôler l'accès aux ressources qui se trouvent sur ce territoire (Dejouhanet, 2007).

30. Cf. Exposé des motifs de la loi n² 2005-019 du 17 octobre 2005 fixant les principes régissant les statuts des terres.

31. Articles 40 et 41 de la loi ${ }^{\circ}$ 97-017 du 8 août 1997 portant révision de la législation forestière (J.O.R.M. n²449 du 25 août 1997, p.1717) et les articles 33 et 34 du décret $n^{\circ}$ 2005-849 du 13 décembre 2005 portant refonte des conditions générales d'application de la loi n ${ }^{\circ}$ 7-017 du 8 août 1997. 
Comme le Protocole renvoie au droit national l'institution des droits des communautés locales liés aux ressources objets d'accès dans le cadre de l'APA, et que ces droits sont reconnus aussi bien par la Constitution que par les conventions internationales ratifiées par Madagascar, le statut des bénéficiaires de ces droits devrait être prévu et amélioré aussi bien par projet de loi APA que dans les contrats de bioprospection. En se référant à la définition donnée par les instruments internationaux, il serait judicieux que le projet de loi et les contrats de bioprospection permettent à ces communautés de donner ou non leur consentement pour l'accès aux ressources génétiques. En outre, pour identifier facilement la communauté concernée par la zone de collecte, il convient de prévoir un mécanisme permettant de déterminer un représentant légitime de la communauté s'exprimant en son nom (Commissariat général au développement durable, 2010).

Ainsi, le flou actuel du statut foncier des usufruitiers et des occupants traditionnels ne devrait pas être une occasion pour limiter leur participation aux prises de décisions, et particulièrement à leur droit au consentement lors de l'accès aux ressources biologiques.

Flou des dispositifs internes garantissant le recueil du consentement préalable et la participation des communautés locales en cas d'accès aux ressources

La procédure d'APA prévoit que le consentement préalable et la participation des communautés locales sont obtenus pour l'accès aux ressources génétiques, dès lors que leur droit d'accorder l'accès à ces ressources est établi, conformément aux dispositions législatives ou réglementaires internes (article 6 et 7 du Protocole). Ainsi, le Protocole renvoie le soin d'application de la participation de la population locale au processus APA à des normes internes.

Pour certains auteurs, le consentement préalable donné en connaissance de cause signifie, au sens très large, que l'on donne son consentement à une activité après avoir reçu l'entière divulgation des raisons qui motivent cette activité, des procédures spécifiques entraînées par cette activité, des risques potentiels qui lui sont associés et de toutes les implications que l’on peut prévoir (Glowka et al, 1996; Karpe, 2013).

La reconnaissance du droit au consentement des communautés locales, détentrices des droits rattachés aux ressources objets d'accès, n'est pas un concept nouveau. Sur le plan international, il jouit d'une reconnaissance croissante et constitue un moyen de mise en œuvre des droits des peuples autochtones et des communautés locales. Ainsi, la Convention africaine pour la conservation de la nature et 
des ressources naturelles, que Madagascar a ratifiée $e^{32}$, vise à " préserver les droits traditionnels et de propriété des communautés locales et exiger le consentement préalable des communautés concernées pour tout ce qui concerne l'accès à et l'utilisation des connaissances traditionnelles ${ }^{33}$. En outre, la Déclaration des Nations Unies sur les droits des peuples autochtones ${ }^{34}$, dans son article 32, paragraphe 2, stipule que « les États [doivent consulter] les populations autochtones et coopérer de bonne foi avec celles-ci afin d’obtenir leur consentement libre et informé préalablement à l'approbation de tout projet affectant leurs terres, territoires ou autres ressources, en particulier lorsqu'il est lié au développement, à l'utilisation [...], de l'eau ou d'autres ressources".

En ce qui concerne le droit à la participation aux prises de décision et au consentement, le cadre juridique malgache relatif à la participation du public au processus décisionnel en matière d'évaluation environnementale (arrêté no 6830/2001) reste imprécis quant au contenu de ces droits. En ce sens, il énonce que la participation du public à lévaluation environnementale consiste à récolter les avis et les opinions du public pour réunir un certain nombre déléments pouvant influencer la prise de décision. La participation consiste à informer le public concerné de l'existence du projet et de recueillir ses avis à ce propos (article 2). Ainsi, ce texte limite la participation des membres du public à lémission de leurs avis et préoccupations par rapport au projet de développement qui peut affecter leurs droits (article 20). Or, dans le cadre du consentement, une communauté a la possibilité de négocier des attentes acceptables, et a le droit d'accorder ou de refuser son consentement, lorsque ses priorités et ses préoccupations ne sont pas adéquatement discutées et prises en compte ${ }^{35}$. La distinction entre les termes « consultation » et « consentement » réside

32. A Madagascar, les Traités sont applicables dès leur ratification : ils ont une position spécifique, qui est en loccurrence supérieure aux lois internes. Ainsi, les normes internationales sont directement applicables aux juridictions nationales et par conséquent aux justiciables. Dans ce cadre, un magistrat peut écarter une norme nationale si celleci contredit une norme internationale ratifiée.

33. Avis juridique de la commission africaine des droits de l'homme et des peuples sur la Déclaration des Nations Unies sur les droits des Peuples Autochtones. Disponible http://www.achpr.org/francais/Special\%20Mechanisms/Indegenous/Advisory\%20 opinion_frn.pdf (consulté le 10 mars 2014).

34. Assemblée générale des Nations Unies, Résolution 61/295 (13 septembre 2007).

35. En ce sens, cf. Document de travail préliminaire sur le principe de consentement préalable donné librement et en connaissance de cause des peuples autochtones concernant des aménagements affectant leurs terres et ressources naturelles, qui serait appelé à servir de cadre à la rédaction par le Groupe de travail d'un commentaire juridique relatif à ce concept, présenté par Antoanella-Iulia Motoc et la Fondation Tebtebba, Doc. off. CES NU, 22e sess. Doc. NU E/CN.4/Sub.2/AC.4/2004/4 au paragraphe 27 [Document préliminaire]. 
essentiellement dans la négociation, c'est-à-dire la façon dont une communauté peut exercer son droit et dans le respect de ce droit par l'État et l'utilisateur des ressources génétiques ${ }^{36}$. En bref, dans le cadre de ce processus, une communauté a le droit de dire non et si elle décide de dire oui, elle peut alors négocier les termes en vertu desquels elle acceptera les changements proposés sur les terres et les ressources dont elle dépend (UN-REDD, 2011). Par ailleurs, cet arrêté précise que, au cours de l'ouverture de l'enquête publique, les enquêteurs ont « la faculté de recueillir les avis des membres de la communauté et des groupements ou associations directement concernés ou intéressés par le projet ${ }^{37}$. Ainsi, si on se contente de ce texte pour évaluer la participation des communautés locales dans le processus APA, les enquêteurs peuvent ne pas considérer les appréciations de ces derniers.

Ainsi, dans le cadre de la mise en œuvre du Protocole, l'arrêté sur participation du public à lévaluation environnementale n’est pas du tout approprié. Dans le cadre de l'APA, les principes du consentement doivent guider la participation de tous les détenteurs des droits liés à l'accès aux ressources biologiques. Ces principes sont applicables dans le processus de négociation d'avantages justes et équitables à chaque étape de l'accès et de l'utilisation des ressources génétiques. Il convient par ailleurs de noter qu'une négociation est un processus complexe, elle s'apprend et doit se préparer attentivement et activement. Pour négocier les avantages dans le processus de prise de décision, les compétences ne sont pas les mêmes pour les utilisateurs et les fournisseurs, ainsi que pour le gouvernement et les communautés locales. Ce problème d'équité dans la négociation se rencontre également dans le cadre des conditions convenues d'un commun accord.

\section{Équité des échanges dans les contrats de bioprospection : espoir ou illusion?}

En vue d'un partage équitable des bénéfices, lors de l'accès aux ressources génétiques associées aux connaissances traditionnelles, l'article $5.2 \mathrm{du}$ protocole fait

36. Cf. Instance permanente sur les questions autochtones, Rapport sur les travaux de l'Atelier international sur le consentement préalable, libre et éclairé et les peuples autochtones, New York, 17-19 janvier 2005. Quatrième session, New York, 16-27 mai 2005. E/C.19/2005/3.

37. L'article 25 de l'arrêté $n^{\circ} 6830 / 2001$, fixant les modalités et les procédures de participation du public à l'évaluation environnementale, énonce que « les enquêteurs tiennent une permanence dans les lieux et aux dates indiqués dans l'avis d'ouverture de l'enquête publique, durant laquelle ils recevront en audience toute personne désirant personnellement émettre un avis sur le projet. Selon des modalités définies dans leurs termes de références, les enquêteurs ont, en outre, la faculté de recueillir les avis des membres de la communauté et des groupements ou associations directement concernés ou intéressés par le projet ». 
obligation aux États parties de prendre des mesures législatives et administratives, à travers des conditions convenues d'un commun accord. En ratifiant le Protocole, le pays a l'obligation d'établir des réglementations et des procédures précises sur la demande et létablissement de conditions convenues d'un commun accord. Létablissement de conditions convenues d'un commun accord revient à dire que le fournisseur et l'utilisateur doivent conclure un accord contractuel. Cet accord prévoit les moyens par lesquels les conditions convenues sont fixées, et le recours à des mécanismes contractuels pour le règlement des différends (Gartforth et al, 2005). Les contrats sont assortis de conditions relatives au traçage et à la surveillance des utilisations faites des ressources génétiques basées sur l'obligation de notification (Young, 2004). En cas de conflit dans la mise en œuvre du contrat, l'article 19, paragraphe 2 du Protocole dispose que chaque partie doit garantir la possibilité de recours auprès de ses tribunaux et garantir la possibilité de recours dans leurs systèmes juridiques (article 18, paragraphe 2). En bref, dans le cadre de la justice distributive, le contrat devrait promouvoir la conservation de la biodiversité, éviter la biopiraterie et contribuer à l'équité des échanges (Bellivier \& Noiville, 2006).

Comme on l'a déjà signalé précédemment, faute de cadre juridique spécifique à l'APA, l'accès aux ressources biologiques de Madagascar se base actuellement sur des contrats de bioprospection.

La question qui se pose est alors de savoir si léquilibre contractuel est respecté lors des échanges des fruits de la collecte des ressources biologiques, particulièrement au profit des populations locales et des détenteurs des connaissances traditionnelles.

Afin de répondre à cette question, l'étude du cas d'un contrat de bioprospection conclu entre deux centres nationaux de recherches malgaches (Centre National de Recherche sur l'Environnement et Centre National de Recherche Pharmaceutique) et le projet "International Cooperative Biodiversity Group » (ICGB) va servir d'illustration. En raison de la confidentialité des clauses du contrat, notre analyse se limite à quelques informations concernées par la présente étude.

Ainsi, à la lecture des termes du contrat, on a pu constater que les populations locales riveraines des ressources ont été marginalisées dans les échanges des ressources. Elles ne sont pas particulièrement visées dans les clauses de celui-ci. Certes, la consultation des partenaires locaux y est prévue, mais en fait, ceux-ci nétaient consultés que pour arrêter les activités destinées à recevoir les fonds de compensation « équitable ». Cette situation peut probablement s'expliquer par le flou de leur statut cité précédemment, le prospecteur étant réticent à contracter avec des entités dépourvues d'existence légale (Morin, 2003 ; Hermitte et al, 2006). Ainsi, ce sont uniquement les parties au contrat (Centres 
nationaux malgaches de recherches et Représentation d'ONG américaines de conservation) qui ont prélevé les avantages à leur profit, étant donné que l'obligation d'un partage des bénéfices n'est pas systématique. Compte tenu du rapport de force peu favorable à ces populations, la question se pose de la valeur de leur consentement (à travers cette consultation prévue dans le contrat) et particulièrement des effets du contrat sur celles-ci (Boisvert, 2002).

Il en est de même pour les gardiens des savoirs traditionnels : la préservation et la rémunération de la connaissance indigène sont prévues dans le préambule du contrat, et particulièrement si cette connaissance est utilisée dans la production de produits commercialement viables. Toutefois, dans les termes du contrat, aucune disposition n'est prévue quant au mécanisme de partage des bénéfices issus de l'utilisation des savoirs traditionnels pouvant être rattachés aux matériels biologiques transférés ${ }^{38}$. La raison peut être probablement le flou de leur statut légal (tout comme celui des populations locales). Cette situation a incité certains auteurs à qualifier ces manœuvres de "biopiraterie légale " (Vandana, 2002 ; Stenton, 2004).

Par ailleurs, il convient de signaler la présence de dispositions incompréhensibles dans ce contrat. Il s'agit particulièrement de la désignation des lois du Commonwealth de la Virginie comme applicable, pour interpréter le contrat. Dans ce cadre, on voit bien que ce contrat a été conclu à l'initiative de la partie la plus forte, et que la partie malgache n'a pas eu la maitrise des connaissances techniques et financières, ni la possibilité de négocier ses avantages. Cette situation reflète l'inégalité flagrante des rapports de force entre la partie malgache et la partie américaine, dans la négociation et la rédaction dudit contrat (Kiss et al, 2004). En effet, comme les États-Unis n’ont pas ratifié la convention sur la diversité biologique, ce pays n'est pas tenu de légiférer sur les questions de l'APA. De plus, la législation de ce pays permet de faciliter la biopiraterie (Stenton, 2004 ; Abdelgawad, 2007). Il est alors douteux que la législation de ce pays puisse garantir la protection la plus étendue des intérêts de l'État malgache et de ses populations, et particulièrement des droits des détenteurs des connaissances traditionnelles. En ce sens, on sait très bien qu'en cas de non-respect des clauses du contrat par la partie américaine, ni les populations locales, ni les détenteurs des connaissances traditionnelles nont les moyens financiers et les connaissances juridiques (surtout de la loi américaine) pour agir en justice. En outre, en cas de demande d'annulation du brevet obtenu illégalement sur leurs connaissances, auprès des offices de brevets, il est pratiquement impossible

38. C'est uniquement dans le préambule du contrat qu'il est fait mention des détenteurs des connaissances traditionnelles et qu'il est énoncé que le «Pays malgache et la Population Locale et Indigène de ce pays possèdent la propriété intellectuelle concernant l'utilisation de certains échantillons ». 
pour la partie malgache d'avoir gain de cause auprès de la juridiction américaine. En effet, le coût de la procédure de révocation de brevets aux États-Unis (Abdelgawad, 2009) est très élevé et la partie malgache n’a pas la maîtrise technique et financière des clauses du contrat.

Enfin, les modalités de contrôles constituent des facteurs d'efficacité du partage des avantages. Or, le pays ne dispose pas de systèmes d'audit pour vérifier si les utilisateurs respectent les conditions stipulées dans le contrat (Boisvert, 2002). Ainsi, il lui serait difficile de demander une réparation judiciaire pour les violations de contrat et l'accès illégal, surtout quand on sait que la plupart des violations ont lieu après que la ressource ait été transférée de Madagascar à un autre système de juridiction (Bellivier, 2006).

Cet exemple de contrat montre le déséquilibre flagrant des rapports de force entre utilisateur et fournisseur. Dans tous les cas, ce type de contrat ne peut garantir une protection des connaissances traditionnelles contre la biopiraterie. Pour atteindre l'objectif d'équité, les pays fournisseurs comme Madagascar doivent se doter d'une capacité de négociation et d'un investissement intellectuel. Pour éviter que ne s'impose la loi de la jungle ou celle du plus fort, et contraindre les utilisateurs à respecter les intérêts du fournisseur et de la population, un cadre légal définissant les modalités et le contenu d'un contrat de bioprospection doit être établi. Toutefois, comme lélaboration d'une loi spécifique à l'APA est relativement longue (un projet de loi a été conçu il y a plus de dix ans à Madagascar), des lignes directrices peuvent servir d'instrument de cadrage des contrats de bioprostection (Bellivier \& Noiville, 2006 ; CIRAD, 2011). Ces lignes directrices devraient inclure notamment une clause sur le règlement des différends, les conditions de partage des avantages, compte tenu également des droits de propriété intellectuelle, les conditions de l'utilisation ultérieure par des tiers et les conditions de changement d'intention. Ces deux dernières conditions sont de la plus grande importance pour le pays (Nijar, 2011). Elles obligeront l'utilisateur à obtenir un nouveau consentement donné en connaissance de cause et/ou à conclure de nouvelles conditions convenues d'un commun accord s'il souhaite transférer les ressources à des tiers ou utiliser les ressources à d'autres fins que celles pour lesquelles l'accès a été accordé.

\section{Conclusion}

Le protocole traite des ressources génétiques pour lesquelles les détenteurs des connaissances traditionnelles bénéficient d'un droit reconnu d'accorder leur accès et de bénéficier des avantages qui en découlent. Or, le statut juridique de ces détenteurs des connaissances traditionnelles est encore flou à Madagascar. En ce sens, les réflexions suivantes méritent dêtre approfondies. Qui sont les dé- 
tenteurs des connaissances traditionnelles ? Est-ce la collectivité, les membres de la collectivité, les familles à l'intérieur d'un groupe ou les individus particuliers ? La protection des connaissances traditionnelles devrait-elle s'appliquer uniquement aux tradipraticiens reconnus légalement ou à d'autres détenteurs de connaissances comme les agriculteurs et les guérisseurs " ombiasy " ? Dans quelle mesure la relation entre le droit coutumier et le droit moderne contribue-elle à l'APA ? Pour répondre à ces différentes questions, des recherches, en collaboration avec d'autres disciplines, s'imposent.

Le succès de ce protocole dépendra de sa mise en œuvre efficace au niveau national. En tant que pays fournisseur des ressources génétiques, et ayant ratifié le protocole de Nagoya, Madagascar a l'obligation de se doter d'une législation spécifique à l'APA. Cette dernière lui permet, notamment, de contrôler l'accès et le partage des ressources génétiques liées aux connaissances traditionnelles.

Or, de tout ce qui précède, il apparaît qu'une législation spécifique à l'APA est relativement complexe. Elle nécessite la contribution de plusieurs disciplines, ainsi que des initiatives de renforcement de capacités, particulièrement pour négocier des conditions convenues d'un commun accord et pour ajouter de la valeur aux ressources génétiques, au lieu simplement de faire office de fournisseurs de produits primaires. Ces limites ne doivent toutefois pas constituer un blocage. En effet, si le pays met longtemps à promulguer un cadre juridique spécifique, l'accès aux ressources pourrait soit ne pas s'améliorer, soit continuer sans que le pays et les détenteurs des connaissances liés à ces ressources n’aient à donner leur consentement préalable en connaissance de cause. L'objectif du protocole (garantir le partage des avantages) sera alors compromis, ce qui aura de lourdes répercussions sur les objectifs de conservation et d'utilisation durable des ressources énoncés dans le protocole (article premier) et dans la CDB (article premier).

En attendant l'adoption d'une loi spécifique à l'APA, des lignes directrices ou un code de conduite peuvent constituer une étape importante permettant d'encadrer les contrats de bioprospection afin que les parties puissent respecter les termes du protocole et les principes de transparence.

\section{Bibliographie}

\section{Ouvrages}

AUBERTIN Catherine, PINTON Florence \& Valérie BOISVERT (sous la dir. de), Les marchés de la biodiversité, Paris, Éditions de l'IRD, 2007, 269 p.

BÉRARD Laurence, CEGARRA Marie, DJAMA Marcel, LOUAFI Sélim, MARCHENAY Philippe, ROUSSEL Bernard \& François VERDEAUX (Sous la dir. de), Biodiversité et savoirs naturalistes locaux en France, Paris, INRA/CIRAD/ IDDRI/IFB, 2005, $271 \mathrm{p}$. 
DELPAS Clara, Chroniques de la biopiraterie : du pillage au partage? Montreuil, Ed. Omniscience, Coll. " La manufacture des idées », 2012, 368 p.

DOSSOU-YOVO, Bonaventure, Laccès aux ressources biologiques dans les rapports Nord-Sud : jeux, enjeux et perspectives de la protection internationale des savoirs autochtones. Paris, Édition L'Harmattan, 2008. 438 p.

SHELTON, Dinah, Techniques et procédures en droit international de l'environnement, Genève, UNITAR, (Programme de formation à l'application du droit international de l'environnement), 1999, $133 \mathrm{p}$.

FLEURENTIN Jacques, PELT, Jean-Marie \& Guy MAZARS (sous la dir. de), LEJOSNE, Jean-Claude (trad.) \& CABALION, Pierre (collab.), Des sources $d u$ savoir aux médicaments du futur: actes du $4^{e}$ congrès européen d'ethnopharmacologie. From the Sources of Knowledge to the Medicines of the Future: Proceedings of the 4th European Congress on Ethnopharmocology, Paris, IRD, 2002, $468 \mathrm{p}$.

GARFORTH, Kathryn, LÓPEZ NORIEGA, Isabel, CABRERA MEDAGLIA, Jorge \& Gabriel R. NEMOGÁ. Overview of the National and Regional Implementation of Access to Genetic Resources and Benefit Sharing. Montréal, Canada: Centre for International Sustainable Development Law, 2005. 103 p.

JOHNSON, Pierre William, Biopiraterie: Quelles alternatives au pillage des ressources naturelles et aux savoirs ancestraux?, Paris, Éditions Charles Léopold Mayer, 2011, $240 \mathrm{p}$.

KISS, Alexandre Charles \& Jean-Pierre BEURIER, Droit International de l'environnement, Paris, Pedone, 2004, $3^{e}$ éd., 369 p.

MOINE-DUPUIS, Isabelle (sous la dir. de), Le médicament et la personne: aspects de droit international, Paris, LexisNexis, Édition Litec, 2007.

Organisation de Coopération et de Développement Économique. Préserver la diversité biologique : les incitations économiques. Paris, Édition OCDE, 1996, $178 \mathrm{p}$.

RARIJAONA, René, Le concept de propriété en droit foncier de Madagascar. Étude de sociologie juridique, Paris, Édition Cujas, coll. "Études malgaches ", 1967, $306 \mathrm{p}$.

VANDANA,Shiva, La biopiraterie ou le pillage de la nature et de la connaissance, Paris, Édition Alias, 2002, 165 p.

\section{Articles}

ABDELGAWAD, Walid, "Les contrats internationaux de bioprospection : moyen de protection de la biodiversité et des savoirs traditionnels ou instrument de biopiraterie ? ", Revue Québécoise de droit international 22.1 (mars 2009), 5385. http://rs.sqdi.org/volumes/221_03_Abdelgawad.pdf. Consulté le 09/03/14.

face aux droits des populations locales sur leurs savoirs traditionnels », http:// halshs.archives-ouvertes.fr/halshs-00110350. Consulté le 09/03/14. 
AKNIN, Audrey, FROGER, Géraldine, GERONIMI, Vincent, MÉRAL, Philippe \& Patrick SCEMBRI (sous la dir. de), « Développement durable : enjeux, regards et perspectives ", Cahier du GEMDEV (Groupement d'Intérêt Scientifique pour l'Étude de la Mondialisation et du Développement) 28 (2002), 82-104.

BELLIVIER, Florence \& Christine NOIVILLE, «1992-2007: Les quinze ans du contrat de bioprospection, un anniversaire en demi-teinte ", Revue des contrats 3 (juillet 2007), 917 et s.

—, "Code de conduite et équité des échanges de ressources biologiques, Idées pour le débat », IDDRI (Institut du développement durable et des relations internationales) 15 (2006), 2-20.

BLANC-PAMARD, Chantal \& Hervé RAKOTO RAMIARANTSOA, " Normes environnementales, transferts de gestion et recompositions territoriales en pays betsileo (Madagascar) », Natures Sciences Sociétés 15.3 (2007), 253-268.

BLED, Amandine, "Accès et partage des avantages : rétrospective et principaux enjeux d'ici 2010 ", Cahier de l'IUED (Institut Universitaire d'Études du Développement), 143-148. www.iddri.org/Publications/.../An_0802_Bled_ Avantages.pdf. Consulté le 03/03/14.

BOISVERT, Valérie, "Les contrats de bioprospection et la question du partage des avantages », AKNIN Audrey, FROGER Géraldine, GERONIMI Vincent, MÉRAL Philippe \& Patrick SCHEMBRI (sous la dir. de), Développement durable : enjeux, regards et perspectives, Cahier du GEMDEV 28 (2002), 82-104.

BUCK, Matthias \& Clare HAMILTON. "The Nagoya Protocol on Access to Genetic Resources and the Fair and Equitable Sharing of Benefits Arising from their Utilization to the Convention on Biological Diversity." Review of European Community \& International Environmental Law (RECIEL) 20.1 (2011): 47-61. http://www.publicgardens.org/files/files/Buck_and_Hamilton_2011_Nagoya_ Protocol.pdf. Consulté le 05/03/14.

CABRERA MEDAGLIA, Jorge \& Christian LOPEZ SILVA, « Répondre aux problèmes de l'accès aux ressources génétiques : protection des sources et certitude pour les utilisateurs », Gland, Suisse, UICN (Union Internationale pour la Conservation de la Nature), 2008, 81 p. http://cmsdata.iucn.org/downloads/ eplp_67_1_fr.pdf. Consulté le 04/03/14.

CARRIZOSA, Santiago, BRUSH, Stephen B., WRIGHT, Brian D., \& Patrick E. MCGUIRE. "Accessing Biodiversity and Sharing the Benefits: Lessons from Implementing the Convention on Biological Diversity." IUCN Environmental Policy and Law Paper 54, IUCN-ELP. 2004, 334 p. https://www.iucn.org/ about/work/programmes/environmental_law/elp_resources/elp_res_publications/?uPubsID=3009, Consulté le 03/03/14. 
CORTADELLAS Dominique, ANDRIANTSIFERANA, Marta, LABOUTE Pierre \& Louisette RAZANAMPARARANY, « Les substances naturelles d'intérêt pharmacologique ». FELLER, Christian \& Frédéric SANDRON (sous la dir. de), Parcours de recherche à Madagascar, Marseille, IRD Éditions, 2010, 391-405.

COSTES, Cyril, « La biopiraterie, les savoirs traditionnels et le droit », Ikewan 67 (janvier-mars, 2008), 3-11.

HERMITTE, Marie-Angèle \& Philippe KHAN (sous la dir. de), "Les ressources génétiques végétales et le droit dans les rapports Nord/Sud ", Revue québécoise de droit international 17.2 (octobre 2005), 303-305.

HERMITTE, Marie-Angèle (coord.), MALJEAN-DUBOIS, Sandrine, NOIVILLE, Christine, MABILE, Sébastien, BELLIVIER Florence \& Isabelle DOUSSAN, «La Convention sur la diversité biologique a quinze ans », Annuaire français de droit international 52.52 (2006), 351-390.

HODGES Timothy \& Anne DANIEL. "Promises and Pitfalls: First Steps on the Road to the International ABS Regime." RECIEL 14.2 (2005), 148-160.

HUFTY, Marc, CHOLLET, Martial \& Andry RAZAKAMANANTSOA, " Néo-colonialisme vert à Madagascar? ", SABELLI, Fabrizio (sous la dir. de), Lécologie contre nature, IUED (1995), 143-148.

KARPE Philippe, "Le droit au consentement préalable, libre et éclairé des collectivités autochtones ", FOATAS 1 : Forêts Africaines-tabernacles des savoirs : Les peuples autochtones et le Libre Consentement Préalable et Éclairé (2013), 51-68.

KISS, Alexandre Charles, "La notion de patrimoine commun de l'humanité », RCADI (Recueil des Cours de l'Académie de Droit International) 175 (1982), 109-253.

LEFÈVRE, Gabriel, « Les discours sur la médecine traditionnelle à Madagascar », Revue des Sciences Sociales, "Éthique et santé » 39 (2008), 46-59.

MACKAY, Fergus, «Le droit des peuples autochtones au libre consentement préalable et éclairé et la Revue des industries extractives de la Banque mondiale-Une synthèse », Forest Peoples Programme (28 juin 2004), 48 p.

MORETTI, Christian \& Catherine AUBERTIN, «Stratégies des firmes pharmaceutiques : la bioprospection en question ", AUBERTIN Catherine, PINTON Florence \& Valérie BOISVERT (sous la dir. de), Les marchés de la biodiversité, Paris, IRD Éditions, 2007, 27-54.

MORIN, Jean-Frédéric, "Les accords de bioprospection répondent-ils aux objectifs de la convention sur la diversité biologique ? ", RDUS (Revue de Droit de l'Université de Sherbrooke) 34.1 (2003), 307-343. https://www.usherbrooke. $\mathrm{ca} / \mathrm{droit} /$ fileadmin/sites/droit/documents/RDUS/volume_34/34-12-morin.pdf. Consulté le 10/03/14. 
MUTTENZER, Frank, "Politiques foncières et pluralisme juridique à Madagascar : La reconnaissance de la tenure coutumière dans l'impasse légale ", Revue TALOHA 19 (29 janvier 2010). Consultable sur http://www.taloha.info/ document.php ?id $=755$.

NEWMAN, David J. \& Gordon M. CRAGG. "Natural Products as Sources of New Drugs over the 30 Years from 1981 to 2010." Journal of Natural Products 75.3 (2012): 311-335.

PÉCHARD, Guy, ANTONA, Martine, AUBERT Sigrid \& Didier BABIN, « Ressources phytogénétiques, contrats et application de la convention biodiversité de Madagascar: une approche prospective ", Bois et forêts des tropiques 284 (2005), 90p.

RAMAROLANTO, Ratiaray, "Laccès à la terre en droit rural malgache ", Revue Internationale de Droit Comparé 41.3 (1989), 637-707.

RAMBININTSAOTRA, Saholy \& Ratiaray RAMAROLANTO, "Laccès coutumier aux ressources liées à la terre : Défendre l’indéfendable? ", Rakoto RAMIARANTSOA, Hervé, BLANC-PAMARD, Chantal \& Florence PINTON (Sous la dir. de), Géopolitique et environnement: Les leçons de l'expérience malgache, Marseille, IRD Éditions, Collection « Objectifs Suds», 2012, 99-122.

ROSENBERG, Dominique, «Le principe de souveraineté des États sur leurs ressources naturelles ", Revue internationale de droit comparé 36.4 (1984), 898901.

STENTON, Gavin. "Biopiracy within the Pharmaceutical Industry: A Stark Illustration of how Abusive, Manipulative and Perverse the Patenting Process can be towards Countries of the South." EIPR (European Intellectual Property Review) 26.1 (2004): 17-26.

\section{Thèses, mémoires}

RAHARINIRINA, Vahinala. "Valorisation économique de la biodiversité par les contrats de bioprospection et la filière huiles essentielles. Le cas de Madagascar ». Thèse de doctorat en science économique, Université de VersaillesSaint-Quentin-en-Yvelines, 2009. 423 p.

\section{Rapports}

ANDRIANIRINA, Ratsialonana Rivo, "Cadre d'analyse de la Gouvernance Foncière à Madagascar ", Rapport final, Observatoire du Foncier, Madagascar, Banque Mondiale. 2011. http://siteresources.worldbank.org/INTLGA/Resources/LGAF_Madagascar2011_rapport_final.pdf. Consulté le 10/03/14.

CIRAD, INRA, IRD, « Lignes directrices pour l'accès aux ressources génétiques et leur transfert. ", septembre 2011, 60 p. http://www.cirad.fr/content/ download/6126/61293/version/2/file/Lignes+directrices+web-V1.pdf. Consulté le 10/03/14. 
Commissariat Général au Développement Durable, « Donner une valeur à l'environnement : la monétarisation, un exercice délicat mais nécessaire », Collection « La Revue » du SEEIDD du CGDD, décembre 2010.

Commission Africaine de Droit de l'Homme et des Peuples. Avis juridique de la Commission Africaine des Droits de l'Homme et des Peuples sur la Déclaration des nations unies sur les droits des peuples autochtones. (Brazzaville, 15-28 novembre 2007), http://www.afrimap.org/english/images/treaty/CADHP-Avisjuridique-autochtones-2007.pdf. Consulté le 13/12/14.

DEJOUHANET, Lucie, Les produits forestiers non ligneux et la gestion de la forêt kéralaise : droit d'usage et droit de contrôle, Institut français de Pondichéry, 2007. http://www. foncier-developpement.fr/publication/les-produits-forestiers-non-ligneux-et-la-gestion-de-la-foret-keralaise-droit-dusage-et-droit-de-controle/. Consulté le10/03/14.

DURAND J.-M., JONCKHEER S., LIVERSAGE, H. \& P. MATHIEU, «Évaluation institutionnelle de la réforme foncière à Madagascar ", Rapport de mission FIDA/FAO, juillet 2011, 42 p. http://landportal.info/sites/default/files/rapport-fida-fao-mada-vfinale.pdf. Consulté le 10/03/14.

DUTFIELD, Graham, "What is Biopiracy?", document présenté à l'Atelier de travail international sur l'APA (Cuernavaca, octobre 2004). http://moderncms. ecosystemmarketplace.com/repository/moderncms_documents/I.3.pdf. Consulté le 07/03/14

Fondation pour la Recherche sur la Biodiversité, «APA dans tous ses états : accès aux ressources génétiques et partage des avantages issus de leur utilisation ». Fiche-clé n ${ }^{\circ}$, décembre 2011 http://www.cnrs.fr/inee/communication/ actus/docs/Fiche_APA.pdf. Consulté le 03/03/14.

- Pertinence et faisabilité d'un dispositif d'accès et de partage des avantages en outre-mer, portant sur les ressources génétiques et connaissances traditionnelles associées, 2010. http://www.developpement-durable.gouv.fr/IMG/pdf/ Synthese-etude-APA-outre-mer-FRB.pdf. Consulté le 06/03/14.

GLOWKA, Lyle, BURHENNE-GUILMIN Françoise \& SYNGE Hughen, collaboration avec Jeffrey A. MCNEELY \& Lothar GÜNDLING, " Guide de la Convention sur la diversité biologique ", Environmental Law and Policy Paper 30, UICN, 1996, https://portals.iucn.org/library/efiles/documents/EPLP030-Fr.pdf. Consulté le 05/03/14.

GREIBER, Thomas, PEÑA MORENO Sonia, ÅHRÉN Mattias, NIETO CARRASCO Jimena, CHEGE KAMAU Evanson, CABRERA MEDAGLIA Jorge, OLIVA Maria Julia \& Frédéric PERRON-WELCH, Natasha ALI \& China WILLIAMS. "An Explanatory Guide to the Nagoya Protocol on Access to genetic-sharing." Environmental Law and Policy Paper 83, UICN, 2012. https:// cmsdata.iucn.org/downloads/an_explanatory_guide_to_the_nagoya_protocol. pdf. Consulté le 09/03/14. 
LAVIGNE DELVILLE, Philippe, MONGBO Roch \& Aurore MANSION (Sous la dir. de), «Vers une gestion foncière communale : stratégies, outils et conditions de réussite ", Actes de latelier d'échanges de pratiques en Afrique de l'Ouest et à Madagascar (Cotonou, 20-25 octobre 2008). Paris, GRET, 2009. $96 \mathrm{p}$.

MEKOUAR, Ali Mohamed, "Le droit de l'environnement dans la Charte Africaine des droits de l'homme et des peuples ", Étude juridique de la FAO, (avril 2001) $20 \mathrm{p}$.

NIJAR, Gurdial Singh, «Le protocole de Nagoya sur l'accès aux ressources génétiques et le partage juste et équitable des avantages découlant de leur utilisation : analyse et mise en oeuvre des options s'offrant aux pays en développement ». Document de recherche. Genève, Centre Sud, Juillet 2011, $57 \mathrm{p}$.

NNADOZIE, Kent \& Robert LETTINGTON. Report on the Legal Status of Genetic Resources in National Law, Including Property Law, Where Applicable, in a Selection of Countries. https://www.cbd.int/doc/meetings/abs/abswg-05/official/abswg-05-05-en.pdf. Consulté le 09/03/14.

Organisation Mondiale de la Propriété Intellectuelle, "Comité intergouvernemental de la propriété intellectuelle relative aux ressources génétiques, aux savoirs traditionnels et au folklore ", 16 ${ }^{e}$ session (Genève, 3-7 mai 2010). Consulté le 09/03/14.

— " "Guide de l'Organisation mondiale de la propriété intellectuelle sur la fixation des savoirs traditionnel » OMPI, 2012. http://www.wipo.int/ export/sites/www/tk/en/resources/pdf/tk_toolkit_draft_fr.pdf. Consulté le 09/03/14.

-, "Propriété intellectuelle et savoirs traditionnels », 16-20 avril 2012, http://www.wipo.int/export/sites/www/freepublications/fr/tk/920/wipo_ pub_920.pdf. Consulté le 07/03/14.

Organisation Mondiale de la Santé, Bureau régional de l'Afrique, Rapport de situation sur la décennie de la médecine traditionnelle dans la région africaine, 2011. http://www.afro.who.int/index.php?option=com_docman\&task=doc_ download\&gid=6768. Consulté le 06/03/14.

RATSIMAMANGA URVERG, Suzanne "Eugenia Jambolana : Madagascar » : Institut Malgache de Recherche Appliquée, Antananarivo. http://tcdc2. undp.org/GSSDAcademy/SIE/Docs/Vol7/Eugenia_Jambolana_Madagascar. pdf.

RUIZ, Manuel \& Isabel LAPEÑA. "A Moving Target: Genetic Resources and Options for Tracking and Monitoring their International Flows." IUCN Environmental Law and Policy Paper 67/3, ABS Series n ${ }^{\circ}$ 3, IUCN-ELP, 2007. www. iucn.org/...resources/elp_res_publications/? Consulté le 09/03/14. 
Service d'Appui à la Gestion de l'Environnement. Inventaire des textes juridiques relatifs à l'accès aux ressources biologiques et au partage des avantages, 2002. http://voahary-salama.org/images/File/SAGE.pdf. Consulté le 09/03/14.

TVEDT, Morten Walloe \& Tomme YOUNG. "Beyond Access: Exploring Implementation of the Fair and Equitable Sharing Commitment in the CBD." IUCN Environmental Law and Policy Paper 67.2, ABS Series $n^{\circ}$ 2. IUCN-ELP, 2007. http://www.iucn.org/about/work/programmes/environmental_law/elp_ resources/elp_res_publications/?uPubsID $=3460$. Consulté le 08/03/14.

TVEDT, Morten Walloe. "Elements for Legislation in User Countries to Meet the Fair and Equitable Benefit Sharing Commitment." Journal of World Intellectual Property 9.2 (march 2006), 189-212. http://www.fni.no/doc\&pdf/ mwt-JWIP-2006.pdf. Consulté le 09/03/14.

UN-REDD, Lignes Directrices sur le Consentement Libre, Informé et Préalable du Programme ONU-REDD, http://www.unredd.net/index.php?option=com docman\&task=doc_download\&gid=6368\&Itemid=53. Consulté le 06/03/14.

YOUNG, Tomme. "Genetic Resources and Utilization of Genetic Resources: a Legislative View." Document présenté à l'Atelier de travail international sur l'APA (Cuernavaca, Mexique, octobre 2004).

\section{Textes juridiques nationaux :}

Loi no 2011-002 du 22 août 2011 portant Code de la Santé.

Loi n ${ }^{\circ}$ 96-025 du 30 septembre 1996 sur la gestion locale des ressources naturelles renouvelables.

Loi $\mathrm{n}^{\circ}$ 90-033 du 21 décembre 1990 modifiée par les lois $\mathrm{n}^{\circ}$ 97-012 du 06.06.1997 et n ${ }^{\circ}$ 2004-015 du 19 août 2004 portant Charte de l'Environnement et ses modificatifs (J.O.R.M. no 2035 du 24.12.90, p. 2540) modifiée par la loi $\mathrm{n}^{\circ}$ 97-012 du 6 juin 1997 (J.O.R.M. du 09.06.97, p. 1171, Édition spéciale et $\mathrm{n}^{\circ} 2584 \mathrm{du} 12.07 .99$, p. 1479).

Ordonnance $\mathrm{n}^{\circ}$ 89-019 du 19 juillet 1989 instituant un régime pour la protection de la propriété industrielle.

Décret n $n^{\circ}$ 99-954 du 15 décembre 1999 relatif à la mise en compatibilité des investissements avec l'environnement modifié.

Décret $\mathrm{n}^{\circ}$ 95-695 du 3 Novembre 1995 portant ratification de la convention sur la diversité biologique.

Arrêté $\mathrm{n}^{\circ} 6830$ du 28 juin 2001 fixant les modalités et les procédures de participation du public à lévaluation environnementale.

Résumé : Madagascar est mondialement réputé pour la richesse de sa biodiversité. Depuis quelques années la propriété anti-leucémique de la pervenche rose de Madagascar a été identifiée. Toutefois le pays et surtout les détenteurs du savoir à l'origine de la découverte de ces caractéristiques ne profitent pas des bénéfices qui en sont issus. L'adoption récente du Protocole de Nagoya sur l'accès et le partage équitable des avantages qui en découlent constitue une opportunité pour éviter cette 
forme de biopiraterie. Madagascar ne dispose pas encore de cadre juridique de mise en œuvre de ce traité même si le pays vient de le ratifier. L'accès aux ressources biologiques se base actuellement sur des contrats bilatéraux. Cet article fait un état des lieux juridique pour un partage équitable des avantages lors de l'accès aux ressources génétiques, et surtout des connaissances traditionnelles qui en découlent. Dans ce cadre, sont étudiées les forces et faiblesses des contrats et des cadres juridiques afin de souligner les facteurs d'évolution et de blocage pour une mise en œuvre effective d'un partage conforme aux objectifs du Protocole.

Mots clés : Ressources biologiques, Accès et partage des avantages, État des lieux juridiques, droits locaux.

Summary: Madagascar is well known throughout the world for its rich biodiversity. A few years ago, the anti-leukemia property of the Madagascar periwinkle (Catharanthus roseus) was discovered. However, the country and above all the owners of the knowledge at the origin of the discovery are not benefiting from it. The recent adoption of the Nagoya Protocol on access and benefit-sharing is an opportunity to prevent this form of biopiracy. Madagascar does not yet have a legal frame within which this treaty can be implemented if the country ratifies it. For now, access to biological resources is based on bilateral contracts. This article makes an assessment of the legal situation as regards the equal sharing of benefits from access to genetic resources, and traditional knowledge related to them. The strengths and weaknesses of contracts and legal frames are examined to highlight the factors of evolution or obstacles for an effective implementation of resource sharing as recommended by the Protocol.

Keywords: Biological resources, Access and Benefit-sharing, Legal update, Local rights.

Saholy H. Rambinintsaotra est maître de conférences en droit public au Département de droit de I'Université d'Antananarivo, Madagascar, et chercheuse associée au Centre de Recherche Interdisciplinaire en Droit de l'Environnement, de l'Aménagement et de l'Urbanisme, Limoges, France. Elle est membre du comité scientifique de la Revue Africaine de Droit de l'Environnement.

Saholy H. Rambinintsaotra is an associate professor in public law at the Law department of the University of Antananarivo, Madagascar, and an associate researcher at the Interdisciplinary Research Center on Environmental Rights, Town and Country Planning (Limoges, France). She is also a member of the scientific committee of the African Journal of Environmental Rights. 